\title{
Approximate capacity of the symmetric half-duplex Gaussian butterfly network
}

\author{
A. Salman Avestimehr \\ California Institute of Technology, \\ Pasadena, California, USA. \\ avestime@caltech.edu
}

\author{
Tracey Ho \\ California Institute of Technology, \\ Pasadena, California, USA. \\ tho@caltech.edu
}

\begin{abstract}
In this paper we study the capacity of the halfduplex wireless butterfly network, in which a relay node facilitates the communication between two interfering transmitterreceiver pairs. We use the deterministic approach to make progress towards approximating the capacity region of this network. We use the insights obtained from the analysis of the corresponding deterministic problem to derive a new upper bound on the capacity of this network. We also propose a transmission strategy and show that for symmetric channel gains the gap between its achievable rate region and the upper bound is at most $\frac{\log 15}{2} \approx 1.95 \mathrm{bits} / \mathrm{sec} / \mathrm{Hz}$ per user.
\end{abstract}

\section{INTRODUCTION}

The wireless butterfly network, shown in Figure 1, is a canonical scenario where network coding improves throughput compared to routing [1]. It is one of the basic coding configurations exploited in COPE [2], a practical network coding protocol shown experimentally to be able to double or triple throughput in 802.11 mesh networks. In this network the source node $S_{i}, i=1,2$ wishes to communicate to the destination node $D_{i}, i=1,2$ simultaneously utilizing the relay $R$. The traditional wireless network coding model assumes unit capacity broadcast links that are operated orthogonally, with error-free decoding on each link. Information from each source is decoded at the relay and the opposite destination. The relay transmits the bitwise XOR of its inputs, from which each destination is able to decode its message using knowledge of the other message.

These and other works highlight scenarios where network coding is useful, though the assumption of separate physical layer and network layer coding is suboptimal. In this paper we study the information theoretic capacity of the wireless butterfly network, with the linear additive Gaussian noise channel model. Motivated by the deterministic approach in [3], here we make progress towards the goal of approximating the capacity region of this network. In a related work [4], authors have proposed and analyzed a few decode-forward relaying strategies for this network (in the presence of a direct link between each source and destination).

The deterministic model simplies the wireless interaction model by eliminating the noise and allows us to focus more on signal interactions. This approach was successfully applied to the relay network in [3], and resulted in insight into transmission techniques which further led to an approximate capacity characterization of the noisy wireless relay network [5]. This approach was also applied recently to the bidirectional relay channel problem [6], which again resulted in approximating its capacity region.

Inspired by these results, we examine the deterministic halfduplex butterfly network. We first derive a new outer bound on its capacity which is tighter than the cut-set upper bound. Then in the case that the channel gains are symmetric, we propose a scheme that achieves the outer bound and characterize the capacity exactly. Quite interestingly, the side channels (i.e. the channel between $S_{1}$ and $D_{2}$ and between $S_{2}$ and $D_{1}$ ) are used for two different purposes: in the first phase (i.e. relay listens) it is used by each destination to decode a part of the interference in the relay's signal. In the second phase (i.e. relay transmits) each source uses the side channel to cancel another part of the interference in the relays's transmitted signal. This transmission technique is called interferenceneutralization and was recently proposed in [7].

Next, we use the insights that we obtained so far and analyze the capacity of the symmetric Gaussian butterfly network. By following similar steps, we first derive an outer bound on its capacity. Then we show that a natural translation of our earlier scheme achieves within $\frac{\log 15}{2} \approx 1.95 \mathrm{bits} / \mathrm{sec} / \mathrm{Hz}$ per user of the upper bound for symmetric channels gains and hence approximate its capacity to within a constant.

\section{System Model}

The system model for the wireless butterfly network is shown in Figure 1. We assume that there is a half-duplex constraint on the relay which means it can not listen and transmit at the same time. We assume that the fraction of the time that the relay is listening is fixed and denoted by $t$. Although $t$ can not adaptively change as a function of the channel gains, one can optimize it beforehand. Therefore as shown in Figure 2 the network has two modes of operation, in the first mode the relay listens and in the second mode it transmits. We denote the transmit and received signal of node $i$ at mode $j(j=1,2)$ by respectively $X_{i, j}$ and $Y_{i, j}$.

In the AWGN channel model we have

$$
\begin{aligned}
Y_{D_{1}, 1} & =h_{S_{1} D_{2}} X_{S_{2}, 1}+Z_{D_{1}, 1} \\
Y_{D_{2}, 1} & =h_{S_{1} D_{2}} X_{S_{1}, 1}+Z_{D_{2}, 1} \\
Y_{R, 1} & =h_{S_{1} R} X_{S_{1}, 1}+h_{S_{2} R} X_{S_{2}, 1}+Z_{R, 1} \\
Y_{D_{1}, 2} & =h_{S_{2} D_{1}} X_{S_{2}, 2}+h_{R D_{1}} X_{R, 2}+Z_{D_{1}, 2} \\
Y_{D_{2}, 2} & =h_{S_{1} D_{2}} X_{S_{1}, 2}+h_{R D_{2}} X_{R, 2}+Z_{D_{2}, 2}
\end{aligned}
$$


where $Z$ 's are i.i.d. complex Gaussian noises with power 1 and $h_{i . j}$ 's are complex numbers representing the channel gains. There is also an average power constraint equal to 1 at each node (in each mode).

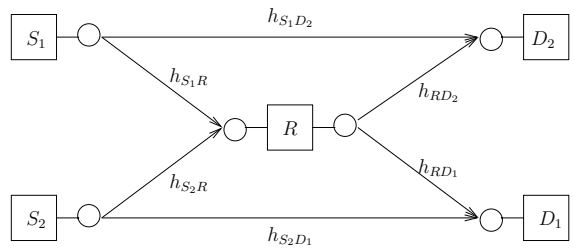

Fig. 1. The system model for wireless butterfly network.

\section{THE DETERMINISTIC BUTTERFLY NETWORK}

In this section we analyze the capacity region of the deterministic half-duplex butterfly network. Here is a formal definition of this model.

Definition 3.1: (Definition of the deterministic model [3]) Consider a wireless network as a set of nodes $V$, where $|V|=$ $N$. Communication from node $i$ to node $j$ has a non-negative integer gain $n_{(i, j)}$ associated with it. This number models the channel gain in a corresponding Gaussian setting. At each time $t$, node $i$ transmits a vector $\mathbf{x}_{i}[t] \in \mathbb{F}_{2}^{q}$ and receives a vector $\mathbf{y}_{i}[t] \in \mathbb{F}_{2}^{q}$ where $q=\max _{i, j}\left(n_{(i, j)}\right)$. The received signal at each node is a deterministic function of the transmitted signals at the other nodes, with the following input-output relation: if the nodes in the network transmit $\mathbf{x}_{1}[t], \mathbf{x}_{2}[t], \ldots \mathbf{x}_{N}[t]$ then the received signal at node $\mathrm{j}, 1 \leq j \leq N$ is:

$$
\mathbf{y}_{j}[t]=\sum_{k=1}^{N} \mathbf{S}^{q-n_{k, j}} \mathbf{x}_{k}[t]
$$

for all $1 \leq k \leq N$, where $\mathbf{S}$ is the $q \times q$ shift matrix and the summation and multiplication is in $\mathbb{F}_{2}$.

Now that we have defined the deterministic channel model we can apply it to the butterfly network network. A pictorial representation of an example of such network with two pairs is shown in Figure 4. In this Figure each little circle represents a signal level and a bit can be sent on it. The transmit and received signal levels are sorted from MSB to LSB from top to bottom. The channel gain between two nodes $i$ and $j$ indicates how many of the first MSB transmitted signal levels of node $i$ are received at destination node $j$. Now as described in the channel model (2), at each received signal level, the receiver gets only the modulo two summation of the incoming bits.

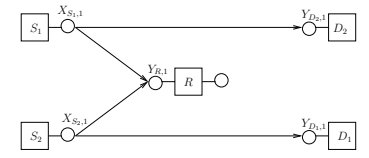

(a) Mode 1

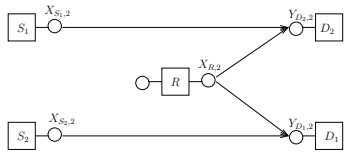

(b) Mode 2
Fig. 2. Network has two modes of operation; in the first node the relay listens (a), in the second mode the relay transmits (b).

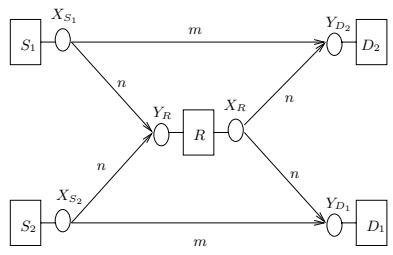

(a) Deterministic

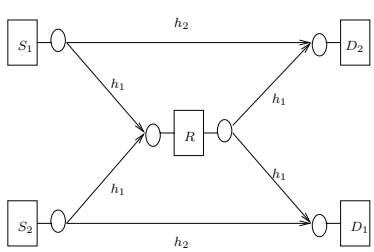

(b) Gaussian
Fig. 3. The symmetric wireless butterfly network

\section{A. Capacity region of the symmetric half-duplex deterministic} butterfly network

Here we examine the case that the channel gains are symmetric. As shown in Figure 3(a) all gains to/from the relay have gain $n$ and the side links have gain $m$. It is easy to show that in this case the optimal duplexing time is $t=0.5$.

Now by applying the cut-set upper bound to this network (with $t=0.5$ ), we obtain the following bound on its capacity region,

$$
R_{1} \leq \frac{n}{2}, \quad R_{2} \leq \frac{n}{2}, \quad R_{1}+R_{2} \leq \frac{m+n}{2}+m .
$$

Now consider the example shown in Figure 4. We note that the rate point $\left(R_{1}, R_{2}\right)=(1.5,1.5)$ is inside the cut-set upper bound region. We ask whether this rate point is achievable?

To answer this question first note that all the information bits that are going from one source to the intended destination are going through the relay. This means that each bit should appear in at least one of the received equations at the relay. Therefore to get a rate of $1.5 \mathrm{bits} / \mathrm{sec}$ per user in the first mode each source should send 3 information bits, as shown in Figure 4 (a). Then, at the end of mode 1, the relay receives the modulo two summation of the bits transmitted by two sources and each destination gets information about the interfering bit on the equation that the relay received at the highest signal level. So if we forward this equation in the second mode, each destination can decode its desired bit from that equation. The side link can also be used to cancel the interference from one of the other two equations, as illustrated in Figure 4 (b). However it does not seem possible to cancel the interfering bit from the remaining equation, hence only one user should use that level. In the next theorem we show that indeed this is true and we derive a genie-aided upper bound on the sum-rate of this network. Furthermore, we also show that the new upper bound is always achievable and hence characterize the capacity region of the symmetric half-duplex deterministic butterfly network.

Theorem 3.2: Consider the half-duplex deterministic butterfly network shown in figure 3(a). The capacity region of this network is characterized by

$0 \leq R_{1} \leq \frac{n}{2}, \quad 0 \leq R_{2} \leq \frac{n}{2}, \quad R_{1}+R_{2} \leq \frac{\max (m, n)}{2}+m$.

Proof:

-Converse: The first two bounds are just based on the cutset upper bound. We just need to prove the bound on the sumrate. Consider any scheme operating over blocks of length $2 T$ ( $T$ time steps in mode 1 and $T$ time steps in mode 2 ), such 


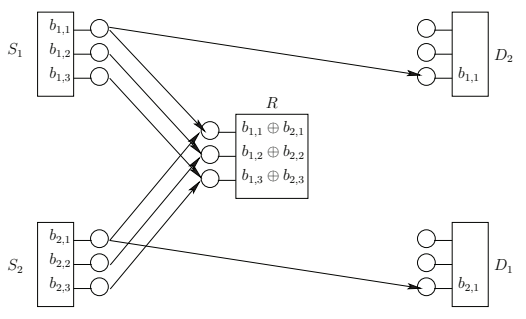

(a) Mode 1

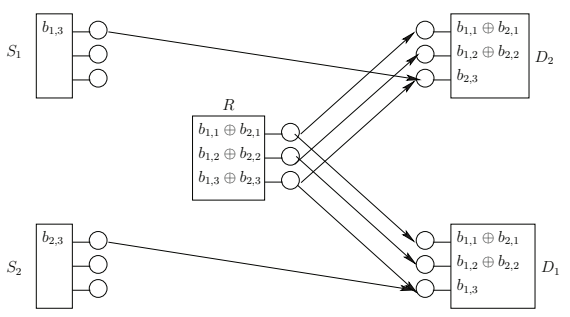

(b) Mode 2

Fig. 4. An example of a symmetric deterministic butterfly network $(n>2 m)$.

that at the end the each destination $D_{i}$ can decode the intended message $W_{i}$ with rate $R_{i}$ with a vanishing error probability $\epsilon_{i, 2 T}, i=1,2$. Now assume that a genie provides $X_{S_{1}, 2}^{T}$ to $D_{2}$. By using data processing and Fano's inequality we have

$2 T\left(R_{1}+R_{2}\right)=H\left(W_{1}\right)+H\left(W_{2}\right)$

$\leq H\left(X_{S_{1}}^{2 T}\right)+H\left(X_{S_{2}}^{2 T}\right)=H\left(X_{S_{1}}^{2 T}, X_{S_{2}}^{2 T}\right)$

$=I\left(X_{S_{1}}^{2 T}, X_{S_{2}}^{2 T} ; Y_{D_{2}}^{2 T}, X_{S_{1}, 2}^{T}\right)+H\left(X_{S_{1}}^{2 T}, X_{S_{2}}^{2 T} \mid Y_{D_{2}}^{2 T}, X_{S_{1}, 2}^{T}\right)$

$=I\left(X_{S_{1}}^{2 T}, X_{S_{2}}^{2 T} ; Y_{D_{2}}^{2 T}, X_{S_{1}, 2}^{T}\right)+H\left(X_{S_{2}}^{2 T} \mid Y_{D_{2}}^{2 T}, X_{S_{1}, 2}^{T}\right)+$

$+H\left(X_{S_{1}}^{2 T} \mid Y_{D_{2}}^{2 T}, X_{S_{1}, 2}^{T} X_{S_{2}}^{2 T}\right)$

$\leq I\left(X_{S_{1}}^{2 T}, X_{S_{2}}^{2 T} ; Y_{D_{2}}^{2 T}, X_{S_{1}, 2}^{T}\right)+H\left(X_{S_{2}}^{2 T} \mid Y_{D_{2}}^{2 T}\right)+$

$+H\left(X_{S_{1}}^{2 T} \mid Y_{D_{2}}^{2 T}, X_{S_{1}, 2}^{T} X_{S_{2}}^{2 T}\right)$

$\leq I\left(X_{S_{1}}^{2 T}, X_{S_{2}}^{2 T} ; Y_{D_{2}}^{2 T}, X_{S_{1}, 2}^{T}\right)+2 T \epsilon_{2,2 T}+H\left(X_{S_{1}}^{2 T} \mid Y_{D_{2}}^{2 T}, X_{S_{1}, 2}^{T} X_{S_{2}}^{2 T}\right)$

$\stackrel{*}{=} I\left(X_{S_{1}}^{2 T}, X_{S_{2}}^{2 T} ; Y_{D_{2}}^{2 T}, X_{S_{1}, 2}^{T}\right)+2 T \epsilon_{2,2 T}+H\left(X_{S_{1}}^{2 T} \mid Y_{D_{1}}^{2 T}\right)$

$\leq I\left(X_{S_{1}}^{2 T}, X_{S_{2}}^{2 T} ; Y_{D_{2}}^{2 T}, X_{S_{1}, 2}^{T}\right)+2 T \epsilon_{2,2 T}+2 T \epsilon_{1,2 T}$

$\leq H\left(Y_{D_{2}}^{2 T}, X_{S_{1}, 2}^{T}\right)+2 T \epsilon_{2,2 T}+2 T \epsilon_{1,2 T}$

$\leq H\left(Y_{D_{2}, 1}^{T}\right)+H\left(Y_{D_{2}, 2}^{T}\right)+H\left(X_{S_{1}, 2}^{T}\right)+2 T \epsilon_{2,2 T}+2 T \epsilon_{1,2 T}$

$\leq T m+T \max (m, n)+T m+2 T \epsilon_{2,2 T}+2 T \epsilon_{1,2 T}$

where $(*)$ is true since given $Y_{D_{2}}^{2 T}$ and $X_{S_{1}, 2}^{T}$, node 2 can reconstruct $Y_{D_{1}}^{2 T}$. Now dividing both sides by $2 T$ and letting $2 T \rightarrow \infty$ we get our bound.

-Achievability: Now we describe the achievability. We consider three cases

Case 1: $m \geq n$

In this case the upper bound is shown in Figure 5 (a). We just need to show that the point $\left(R_{1}, R_{2}\right)=\left(\frac{n}{2}, \frac{n}{2}\right)$ is achievable. The achievability scheme in this case is described below.

1) In mode 1 , source 1 and 2 broadcast $n$ fresh bits,

$$
X_{i, 1}=\left[\begin{array}{llllll}
b_{i, 1} & b_{i, 2} & \ldots & b_{i, n} & 0 & \ldots
\end{array}\right]^{t}, \quad i=1,2
$$

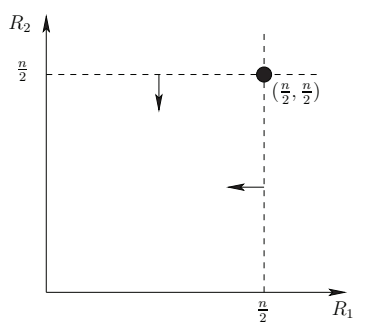

(a) Case 1 and $2\left(m \geq \frac{n}{2}\right)$

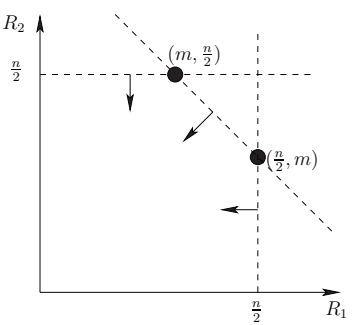

(b) Case $3\left(\frac{n}{2}>m\right)$
Fig. 5. Illustration of the outer-bound; in case 1 and 2 the sum-rate constraint is not active (a), in the third case the sum-rate constraint becomes active (b).

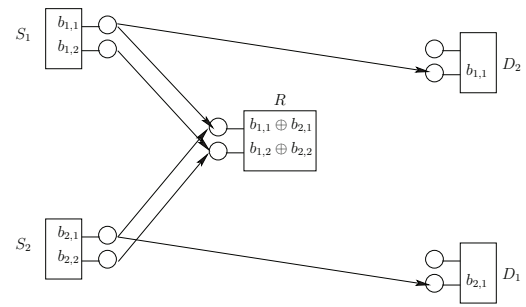

(a) Mode 1

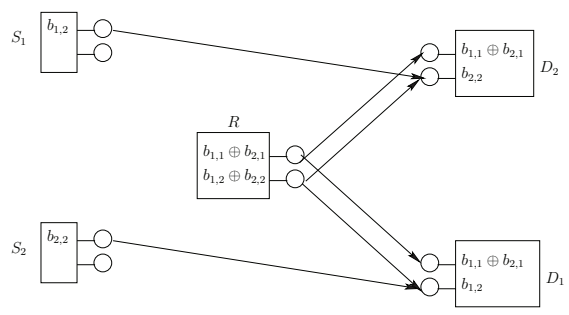

(b) Mode 2

Fig. 6. Illustration of the achievability strategy in case $2(2 m \geq n>m)$.

since $m \geq n$, at the end of the first mode, $D_{1}$ decodes $X_{2,1}$ and $D_{2}$ decodes $X_{1,1}$ and the relay receives

$$
Y_{R, 1}=\left[\begin{array}{llll}
b_{1,1} & b_{1,2} & \ldots & b_{1, n}
\end{array}\right]^{t} \oplus\left[\begin{array}{llll}
b_{2,1} & b_{2,2} & \ldots & b_{2, n}
\end{array}\right]^{t}
$$

2) In mode 2 , both sources are silent and the relay sends $X_{R, 2}=Y_{R, 1}$ and the destinations receive

$$
Y_{1,2}=Y_{2,2}=\left[\begin{array}{lll}
b_{1,1} & \ldots & b_{1, n}
\end{array}\right]^{t} \oplus\left[\begin{array}{lll}
b_{2,1} & \ldots & b_{2, n}
\end{array}\right]^{t}
$$

Now since destination $D_{1}$ had decoded $X_{2,1}$ in the first mode, it knows $\left[\begin{array}{llll}b_{2,1} & b_{2,2} & \ldots & b_{2, n}\end{array}\right]^{t}$ and can decode $\left[\begin{array}{llll}b_{1,1} & b_{1,2} & \ldots & b_{1, n}\end{array}\right]^{t}$. Similarly destination $D_{2}$ can also decode $\left[\begin{array}{llll}b_{2,1} & b_{2,2} & \ldots & b_{2, n}\end{array}\right]^{t}$. Therefore we send $n$ bits from each source to the corresponding destination in 2 time steps and we achieve rate point $\left(R_{1}, R_{2}\right)=\left(\frac{n}{2}, \frac{n}{2}\right)$.

Case 2: $2 m \geq n>m$

The upper bound in this case is the same as before (shown in Figure 5 (a)) and again we just need to show that the point $\left(R_{1}, R_{2}\right)=\left(\frac{n}{2}, \frac{n}{2}\right)$ is achievable. The scheme is pictorially illustrated in Figure 6 and it is more precisely described below.

1) As shown in Figure 6, transmission in mode 1 is the same as before. However, at the end of this mode each destination only decodes the first $m$ bits of the interfering source. 
2) In mode 2 , both sources repeat the last $m$ bits that they sent in previous mode and the relay just forwards its received signal, $X_{R, 2}=Y_{R, 1}$. Now the signal sent by each source cancels the interference in the last $m$ equations coming from the relay and each destination receives the last $m$ bits of its corresponding source. This transmission technique is called interference-neutralization and it was recently proposed in [7]. However at the end of last mode, each destination could also decode the interference in the first $m$ equations coming from the relay. Therefore it can also decode the first $m$ bits coming from its corresponding source. Now since $2 m \geq n$, each destination can decode all $n$ bits sent by the corresponding source and we achieve rate point $\left(R_{1}, R_{2}\right)=\left(\frac{n}{2}, \frac{n}{2}\right)$.

Case 3: $n>2 m$

In this case the upper bound is shown in Figure 5 (b). We need to show that points $\left(R_{1}, R_{2}\right)=\left(\frac{n}{2}, m\right)$ and $\left(R_{1}, R_{2}\right)=$ $\left(m, \frac{n}{2}\right)$ are achievable. The scheme that achieves the first corner point is pictorially illustrated in Figure 4. The scheme is the same as the previous case, except now each destination can cancel interference from only $2 m<n$ equations. Hence the middle levels at the relay are just only used for one sourcedestination pair and one user gets maximum rate $\frac{n}{2}$ and the other one gets rate $m$.

\section{SyMmetric GAUSSIAN BUTTERFLy NETWORK}

In this section we use the intuitions obtained so far to find the approximate capacity region of the symmetric Gaussian butterfly network shown in Figure 3(b). The relationship between the transmit and received signals in this network was described in Section II. We first state our main result,

Theorem 4.1: Consider the half-duplex Gaussian butterfly network shown in figure 3(b) and described in (1). Assume that the relay listen-transmit time is $t=0.5$. The capacity region of this network is within $\frac{1}{2} \log 15 \approx 1.95 \mathrm{bits} / \mathrm{sec} / \mathrm{Hz}$ per user of the following region

$$
\begin{gathered}
0 \leq R_{1} \leq \frac{1}{2} \log \left(1+\left|h_{1}\right|^{2}\right), \quad 0 \leq R_{2} \leq \frac{1}{2} \log \left(1+\left|h_{1}\right|^{2}\right) \\
R_{1}+R_{2} \leq \frac{1}{2} \log \left(\left(1+\left|h_{2}\right|^{2}\right)\left(2+\left|h_{2}\right|^{2}\right)\left(1+\left|h_{1}\right|^{2}+\left|h_{2}\right|^{2}\right)\right)
\end{gathered}
$$

\section{Proof:}

-Converse: See Appendix A.

-Achievability: Now we describe the achievability strategy. Similar to the deterministic case we consider three cases. However due to the limitation of the space we only discuss the last case which is more general than the other ones.

Assume $\left|h_{1}\right| \geq\left|h_{2}\right|^{2} \geq 1$. We want to show that we can achieve within $\frac{1}{2} \log 15$ bits per user of the following

$$
\begin{aligned}
& R_{1}^{*}=\frac{1}{2} \log \left(1+\left|h_{1}\right|^{2}\right) \\
& R_{2}^{*}=\frac{1}{2} \log \left(\frac{\left(1+\left|h_{2}\right|^{2}\right)\left(2+\left|h_{2}\right|^{2}\right)\left(1+\left|h_{1}\right|^{2}+\left|h_{2}\right|^{2}\right)}{1+\left|h_{1}\right|^{2}}\right)
\end{aligned}
$$

Motivated by the optimal scheme that we found for the deterministic case, in the first mode each source uses a superposition of three code-words to create the transmit signal,

$$
X_{S_{i}, 1}^{T}=\sqrt{1-\alpha^{2}-\beta^{2}} U_{S_{i}}^{T}+\alpha V_{S_{i}}^{T}+\beta W_{S_{i}}^{T} \quad, i=1,2
$$

where $U\left(S_{i}, 1\right)^{T}, V\left(S_{i}, 1\right)^{T}$ and $W\left(S_{i}, 1\right)^{T}$ are just random Gaussian codewords with variance 1 . Now based on the optimal scheme in the deterministic case, $\alpha$ and $\beta$ are set such that

1) $V\left(S_{i}, 1\right)^{T}$ and $W\left(S_{i}, 1\right)^{T}$ are arrived at noise level at the destinations (i.e. $\alpha^{2}+\beta^{2}=\frac{1}{\left|h_{2}\right|^{2}}$ ),

2) $W\left(S_{i}, 1\right)^{T}$ arrives at the relay with the same power level as the SNR of the side-link (i.e. $\beta=\frac{\left|h_{2}\right|}{\left|h_{1}\right|}$ ).

This results in the following choice for $\alpha$ and $\beta$,

$$
\beta=\frac{\left|h_{2}\right|}{\left|h_{1}\right|}, \quad \alpha=\sqrt{\frac{1}{\left|h_{2}\right|^{2}}-\frac{\left|h_{2}\right|^{2}}{\left|h_{1}\right|^{2}}}
$$

Then at the end of the first mode, the destination nodes and the relay receive

$$
\begin{aligned}
Y_{D_{1}, 1}^{T} & =h_{2}\left(\sqrt{1-\alpha^{2}-\beta^{2}} U_{S_{2}}^{T}+\alpha V_{S_{2}}^{T}+\beta W_{S_{2}}^{T}\right)+Z_{D_{1}, 1}^{T} \\
Y_{D_{2}, 1}^{T} & =h_{2}\left(\sqrt{1-\alpha^{2}-\beta^{2}} U_{S_{1}}^{T}+\alpha V_{S_{1}}^{T}+\beta W_{S_{1}}^{T}\right)+Z_{D_{2}, 1}^{T} \\
Y_{R, 1}^{T} & =h_{1}\left(X_{S_{1}, 1}^{T}+X_{S_{2}, 1}^{T}\right)+Z_{R, 1}^{T}
\end{aligned}
$$

Now each destination attempts to decode the $U$ codeword that it observes by treating $V$ and $W$ as noise. This can be done with low error probability if

$R_{U_{i}} \leq \log \left(1+\frac{\left|h_{2}\right|^{2}\left(1-\alpha^{2}-\beta^{2}\right)}{\left|h_{2}\right|^{2}\left(\alpha^{2}+\beta^{2}\right)+1}\right)=\log \left(1+\frac{\left|h_{2}\right|^{2}-1}{2}\right)$

In the second mode the relay will just adjust the power of the received signal and forward it, while each destination node attempts to send a signal to cancel the interfering $W$ codeword from the relays transmitted signal. More precisely

$$
\begin{aligned}
X_{S_{i}, 2}^{T} & =-\frac{\beta h_{1}^{2}}{h_{2} \sqrt{2\left|h_{1}\right|^{2}+1}} W_{S_{i}}^{T}=-\frac{\left|h_{2}\right| h_{1}^{2}}{h_{2}\left|h_{1}\right| \sqrt{2\left|h_{1}\right|^{2}+1}} W_{S_{i}}^{T}, i=1,2 \\
X_{R, 2}^{T} & =\frac{1}{\sqrt{2\left|h_{1}\right|^{2}+1}} Y_{R, 1}^{T}
\end{aligned}
$$

Note that since $\frac{\left|h_{1}\right|^{4}\left|h_{2}\right|^{2}}{\left(2\left|h_{1}\right|^{2}+1\right)\left|h_{1}\right|^{2}\left|h_{2}\right|^{2}}<\frac{\left|h_{1}\right|^{4}\left|h_{2}\right|^{2}}{2\left|h_{1}\right|^{4}\left|h_{2}\right|^{2}}=\frac{1}{2}<1$, then we are satisfying the power constraint at each source. Now at the second mode destination 1 receives

$$
\begin{aligned}
& Y_{D_{1}, 2}^{T}=h_{1} X_{R, 2}^{T}+h_{2} X_{S_{2}, 2}^{T}+Z_{D_{1}, 2}^{T} \\
& =\frac{h_{1}}{\sqrt{2\left|h_{1}\right|^{2}+1}}\left(\left(h_{1} \sqrt{1-\alpha^{2}-\beta^{2}}\right)\left(U_{S_{1}}^{T}+U_{S_{2}}^{T}\right)+\frac{h_{1}\left|h_{2}\right|}{\left|h_{1}\right|} W_{S_{1}}^{T}\right. \\
& \left.\quad+\alpha h_{1}\left(V_{S_{1}}^{T}+V_{S_{2}}^{T}\right)+Z_{R, 1}^{T}\right)+Z_{D_{1}, 2}^{T}
\end{aligned}
$$

Now since in the previous mode destination 1 was able to decode $U_{S_{2}}^{T}$, it can cancel it from its received signal and create

$$
\begin{aligned}
& \tilde{Y}_{D_{1}, 2}^{T}=\frac{h_{1}}{\sqrt{2\left|h_{1}\right|^{2}+1}}\left(\left(h_{1} \sqrt{1-\alpha^{2}-\beta^{2}}\right) U_{S_{1}}^{T}+\frac{h_{1}\left|h_{2}\right|}{\left|h_{1}\right|} W_{S_{1}}^{T}\right. \\
& \left.\quad+\alpha h_{1}\left(V_{S_{1}}^{T}+V_{S_{2}}^{T}\right)+Z_{R, 1}^{T}\right)+Z_{D_{1}, 2}^{T}
\end{aligned}
$$


Now it will first attempt to decode $U_{S_{1}}^{T}$ by treating $V_{S_{1}}^{T}$ and $W_{S_{1}}^{T}$ as noise. This can be with small error probability if

$$
\begin{aligned}
R_{U_{1}} & \leq \log \left(1+\frac{\left|h_{1}\right|^{2}\left(1-\alpha^{2}-\beta^{2}\right)}{\left|h_{2}\right|^{2}+2 \alpha^{2}\left|h_{1}\right|^{2}+3+\left|h_{1}\right|^{-2}}\right) \\
& =\log \left(1+\frac{\left|h_{2}\right|^{2}-1}{2+3 \frac{\left|h_{2}\right|^{2}}{\left|h_{1}\right|^{2}}+\frac{\left|h_{2}\right|^{2}}{\left|h_{1}\right|^{4}}-\frac{\left|h_{2}\right|^{4}}{\left|h_{1}\right|^{2}}}\right)
\end{aligned}
$$

Since $\left|h_{2}\right| \geq 1$, we have

$$
\log \left(1+\frac{\left|h_{2}\right|^{2}-1}{2+3 \frac{\left|h_{2}\right|^{2}}{\left|h_{1}\right|^{2}}+\frac{\left|h_{2}\right|^{2}}{\left|h_{1}\right|^{4}}-\frac{\left|h_{2}\right|^{4}}{\left|h_{1}\right|^{2}}}\right) \geq \log \left(1+\frac{\left|h_{2}\right|^{2}-1}{5}\right)
$$

Therefore by (11), (15) and (16) it is sufficient to have

$$
R_{U_{1}} \leq \log \left(1+\frac{\left|h_{2}\right|^{2}-1}{5}\right)
$$

Now after decoding $U_{S_{1}}^{T}$, destination $D_{1}$ attempts to decode both $V_{S_{1}}^{T}$ and $V_{S_{2}}^{T}$ by treating $W_{S_{1}}^{T}$ as noise. This can also be done with low error probability if,

$$
\begin{aligned}
R_{V_{i}} & \leq \log \left(1+\frac{\alpha^{2}\left|h_{1}\right|^{2}}{3+\left|h_{2}\right|^{2}+\left|h_{1}\right|^{-2}}\right), \quad i=1,2 \\
R_{V_{1}}+R_{V_{2}} & \leq \log \left(1+2 \frac{\alpha^{2}\left|h_{1}\right|^{2}}{3+\left|h_{2}\right|^{2}+\left|h_{1}\right|^{-2}}\right)
\end{aligned}
$$

Finally, after decoding $V_{S_{1}}^{T}$ and $V_{S_{2}}^{T}$ and removing $V_{S_{1}}^{T}+V_{S_{2}}^{T}$ from $\tilde{Y}_{D_{1}, 2}^{T}$, destination $D_{1}$ attempts to decode $W_{S_{1}}^{T}$. This can be done with low error probability if

$$
R_{W_{1}} \leq \log \left(1+\frac{\left|h_{2}\right|^{2}}{3+\left|h_{1}\right|^{-2}}\right)
$$

Now we set,

$$
R_{V_{1}}=\log \left(1+\frac{\left|h_{1}\right|^{2}-\left|h_{2}\right|^{4}}{\left|h_{2}\right|^{2}\left(3+\left|h_{2}\right|^{2}+\left|h_{1}\right|^{-2}\right)}\right), \quad R_{V_{2}}=0
$$

Hence from (17) and (19) we achieve the following rate from $S_{1}$ to $D_{1}$,

$$
\begin{aligned}
R_{1} & =\frac{1}{2}\left(R_{U_{1}}+R_{V_{1}}+R_{W_{1}}\right) \\
& =\frac{1}{2}\left(\log \frac{\left(\left|h_{2}\right|^{2}+4\right)\left(\left|h_{1}\right|^{2}+3\left|h_{2}\right|^{2}+\left|h_{2}\right|^{2}\left|h_{1}\right|^{-2}\right)}{5\left|h_{2}\right|^{2}\left(3+\left|h_{1}\right|^{-2}\right)}\right) \\
\stackrel{\left(\left|h_{2}\right| \geq 1\right)}{>} & \frac{1}{2} \log \left(\frac{1+\left|h_{1}\right|^{2}}{15}\right)=R_{1}^{*}-\frac{1}{2} \log 15
\end{aligned}
$$

We can also achieve the following rate from $S_{2}$ to $D_{2}$,

$$
\begin{aligned}
& R_{2}=\frac{1}{2}\left(R_{U_{2}}+R_{V_{2}}+R_{W_{2}}\right) \\
& =\frac{1}{2} \log \left(\frac{\left(\left|h_{2}\right|^{2}+4\right)\left(3+\left|h_{2}\right|^{2}+\left|h_{1}\right|^{-2}\right)}{5\left(3+\left|h_{1}\right|^{-2}\right)}\right) \\
& \left(\left|h_{1}\right|>\left|h_{2}\right|^{2} \geq 1\right) \frac{1}{2} \log \left(\frac{1}{15}\left(1+\left|h_{2}\right|^{2}\right)\left(2+\left|h_{2}\right|^{2}\right) \frac{1+\left|h_{1}\right|^{2}+\left|h_{2}\right|^{2}}{1+\left|h_{1}\right|^{2}}\right) \\
& =R_{2}^{*}-\frac{1}{2} \log 15
\end{aligned}
$$

Therefore we achieve within $\frac{1}{2} \log 15$ bits per user of the desired corner point. Similarly we can show this for the other corner point. Therefore we are at most $\frac{1}{2} \log 15$ bits per user away from the upper bound.

\section{REFERENCES}

[1] Y. E. Sagduyu and A. Ephremides, "Joint scheduling and wireless network coding," Proc. 1st Workshop on Network Coding, Theory and Applications, 2005.

[2] S. Katti, D. Katabi, Wenjun Hu and and Rahul Hariharan, "The Importance of Being Opportunistic: Practical Network Coding For Wireless Environments," Proc. 43rd Allerton Conf.Commun. Contr. Comput., Monticello, IL, 2005.

[3] A. S. Avestimehr, S. N. Diggavi, and D. Tse, "A deterministic approach to wireless relay networks," Proc. Forty-fifth Allerton Conf. Commun. Contr. Comput., Illinois, Sep 2007.

[4] O. Sahin and E. Erkip, "Achievable rates for the Gaussian interference relay channel," Proc. GLOBECOM Communication Theory Symposium, Washington D.C., November 2007.

[5] A. S. Avestimehr, S. N. Diggavi, and D. Tse, "Approximate capacity of gaussian relay networks ," Proc. IEEE ISIT, Toronto, July 6-11, 2008.

[6] S. Avestimehr, A.Sezgin, and D. Tse, "Approximate capacity of the two-way relay channel: A deterministic approach," Proc. Forty-sixth Allerton Conf.Commun. Contr. Comput., Illinois, Sep 2008.

[7] S. Mohajer, S. Diggavi, C. Fragouli and D. Tse, "Transmission Techniques for Relay-Interference Networks," Proc. Forty-sixth Allerton Conf.Commun. Contr. Comput., Illinois, Sep 2008.

\section{APPENDIX A}

PROOF OF THE CONVERSE FOR THEOREM 4.1

The first two inequalities are from the cut-set bound. Now we prove the third constraint. Consider any scheme operating over blocks of length $2 T$, such that at the end each destination $D_{i}$ can decode the intended message $W_{i}$ with rate $R_{i}$ with a vanishing error probability $\epsilon_{i, 2 T}, i=1,2$. Now assume that a genie provides a side information $s$ to $D_{2}$, where

$$
s=h_{2} X_{S_{1}, 2}^{T}+Z_{D_{2}, 2}^{T}-Z_{D_{1}, 2}^{T}
$$

Now if node $D_{2}$ can decode $X_{S_{2}}^{2 T}$, it can reconstruct

$$
\begin{aligned}
\tilde{Y}_{D_{2}, 2}^{T} & =h_{2} X_{S_{2}, 2}^{T}+Y_{D_{2}, 2}^{T}-s \\
& =h_{2} X_{S_{2}, 2}^{T}+h_{1} X_{R, 2}^{T}+Z_{D_{1}, 2}^{T}=Y_{D_{1}, 2}^{T}
\end{aligned}
$$

Now similar to the deterministic case we can write

$$
\begin{aligned}
2 T & \left(R_{1}+R_{2}\right)=H\left(W_{1}\right)+H\left(W_{2}\right) \leq H\left(X_{S_{1}}^{2 T}\right)+H\left(X_{S_{2}}^{2 T}\right) \\
= & H\left(X_{S_{1}}^{2 T}, X_{S_{2}}^{2 T}\right)=I\left(X_{S_{1}}^{2 T}, X_{S_{2}}^{2 T} ; Y_{D_{2}}^{2 T}, s\right)+H\left(X_{S_{1}}^{2 T}, X_{S_{2}}^{2 T} \mid Y_{D_{2}}^{2 T}, s\right) \\
= & I\left(X_{S_{1}}^{2 T}, X_{S_{2}}^{2 T} ; Y_{D_{2}}^{2 T}, s\right)+H\left(X_{S_{2}}^{2 T} \mid Y_{D_{2}}^{2 T}, s\right)+H\left(X_{S_{1}}^{2 T} \mid Y_{D_{2}}^{2 T}, s, X_{S_{2}}^{2 T}\right) \\
& (23) \\
\stackrel{=}{=} & I\left(X_{S_{1}}^{2 T}, X_{S_{2}}^{2 T} ; Y_{D_{2}}^{2 T}, s\right)+H\left(X_{S_{2}}^{2 T} \mid Y_{D_{2}}^{2 T}, s\right)+ \\
& +H\left(X_{S_{1}}^{2 T} \mid Y_{D_{2}}^{2 T}, s, X_{S_{2}}^{2 T}, Y_{D_{1}, 2}^{T}\right) \\
\leq & I\left(X_{S_{1}}^{2 T}, X_{S_{2}}^{2 T} ; Y_{D_{2}}^{2 T}, s\right)+H\left(X_{S_{2}}^{2 T} \mid Y_{D_{2}}^{2 T}\right)+H\left(X_{S_{1}}^{2 T} \mid X_{S_{2}}^{2 T}, Y_{D_{1}, 2}^{T}\right) \\
\leq & I\left(X_{S_{1}}^{2 T}, X_{S_{2}}^{2 T} ; Y_{D_{2}}^{2 T}, s\right)+H\left(X_{S_{2}}^{2 T} \mid Y_{D_{2}}^{2 T}\right)+H\left(X_{S_{1}}^{2 T} \mid Y_{D_{1}, 1}^{T}, Y_{D_{1}, 2}^{T}\right) \\
= & I\left(X_{S_{1}}^{2 T}, X_{S_{2}}^{2 T} ; Y_{D_{2}}^{2 T}, s\right)+H\left(X_{S_{2}}^{2 T} \mid Y_{D_{2}}^{2 T}\right)+H\left(X_{S_{1}}^{2 T} \mid Y_{D_{1}}^{2 T}\right) \\
\leq & I\left(X_{S_{1}}^{2 T}, X_{S_{2}}^{2 T} ; Y_{D_{2}}^{2 T}, s\right)+2 T\left(\epsilon_{1,2 T}+\epsilon_{2,2 T}\right) \\
= & h\left(Y_{D_{2}}^{2 T}, s\right)-h\left(Y_{D_{2}}^{2 T}, s \mid X_{S_{1}}^{2 T}, X_{S_{2}}^{2 T}\right)+2 T\left(\epsilon_{1,2 T}+\epsilon_{2,2 T}\right) \\
\leq & h\left(Y_{D_{2}}^{2 T}, s\right)-h\left(Y_{D_{2}}^{2 T}, s \mid X_{S_{1}}^{2 T}, X_{S_{2}}^{2 T}, X_{R}^{2 T}\right)+2 T\left(\epsilon_{1,2 T}+\epsilon_{2,2 T}\right) \\
= & h\left(Y_{D_{2}}^{2 T}, s\right)-3 T \log 2 \pi e+2 T\left(\epsilon_{1,2 T}+\epsilon_{2,2 T}\right) \\
\leq & h\left(Y_{D_{2}, 1}^{T}\right)+h\left(Y_{D_{2}, 2}^{T}\right)+h(s)-3 T \log 2 \pi e+2 T\left(\epsilon_{1,2 T}+\epsilon_{2,2 T}\right) \\
\leq & T\left(\log \left(2 \pi e\left(1+\left|h_{2}\right|^{2}\right)\right)+\log \left(2 \pi e\left(1+\left|h_{1}\right|^{2}+\left|h_{2}\right|^{2}\right)\right)+\right. \\
& \left.+\log \left(2 \pi e\left(2+\left|h_{2}\right|^{2}\right)\right)\right)-3 T \log 2 \pi e+2 T\left(\epsilon_{1,2 T}+\epsilon_{2,2 T}\right) \\
= & T \log \left(\left(1+\left|h_{2}\right|^{2}\right)\left(2+\left|h_{2}\right|^{2}\right)\left(1+\left|h_{1}\right|^{2}+\left|h_{2}\right|^{2}\right)\right)+2 T\left(\epsilon_{1,2 T}+\epsilon_{2,2 T}\right)
\end{aligned}
$$

Now by diviing both sides by $2 T$ and letting $2 T \rightarrow \infty$ we get our bound. 